This article was published as

Journal of Nanofluids Vol. 7, pp. 1-11, 2018

"Rheological properties of clay suspensions treated by hydrocyclone process".

DOI:10.1166/jon. 2018.1460

\title{
Rheological properties of clay suspensions treated by hydrocyclone process
}

Mariem Mekni Abrougui, ${ }^{a, b}$ Ana Belén Bonhome-Espinosac, Dhouha Bahrid ${ }^{\mathrm{d}}$, Modesto T. López-López, ${ }^{{ }^{*}}$ Juan de Dios García L. Durán ${ }^{c}$, Ezzeddine Srasra ${ }^{a}$

${ }^{a}$ Centre National des Recherches en Sciences des Materiaux. Technopole Borej Cedria, BP 73, 8027

Soliman. Tunisie.

${ }^{b}$ Faculté des Sciences de Tunis, Université de Tunis El Manar, Tunisie B.P. $n^{\circ} 94$ - ROMMANA ‘Tunis 1068

' Department of Applied Physics, Faculty of Sciences, University of Granada, c/ Fuentenueva s/n, 18071, Spain.

d Office National des Mines,17, rue du Cuir - ZI Sidi Rezig - 2033 Megrine. Ben Arous Tunis. Tunisie.

${ }^{*}$ Corresponding author:

Modesto T. López-López, email modesto@ugr.es 


\section{Abstract}

Suspensions of bentonite clays are usually employed at industrial scale in different processes as drilling fluids as well as adsorbents for removing pollutants in muds or natural waters. For these purposes, avoiding the gravitational settling of the particles is a requirement for achieving a high efficiency and a low cost operation. Unfortunately, the clays in natural deposits are usually mixed with particles of other minerals with similar density, making difficult the separation process by usual gravitational methods. Among the most efficient and lowest cost processes, the separation by hydrocyclone is preferred because of a number of advantages at the industrial scale. In this work we verify, by different experimental methods, the efficiency of this wet separation process for removing impurities in a raw bentonite mineral, and at the same time to transform a calcium bentonite in a sodium one by dissolving sodium carbonate in the liquid phase of the hidrocyclone. Afterwards, we checked by using rheological measurements the best protocol for the preparation of the suspension. We studied the rheological behaviour of clay suspensions, with different degree of impurities removal and with different solid concentration, in order to determine the minimal conditions for obtaining bentonite suspensions that do not suffer from gravitational settling during a long period of time. For this purpose, we investigated the deformation and flow of different suspensions, under steady state and oscillatory shear, and determined when they developed a high enough yield stress and an appropriate elastic response to avoid particle settling. We explain the results in view of the energy of interaction between the different surfaces (faces, edges) of the clay platelets, which favours the formation of a soft gel in which the particles are entrapped in loose flocculi that extent along all the volume of the suspensions. 
Key words: Clay, bentonite, hydrocyclone, rheology, viscosity, viscoelasticity.

\section{Introduction}

In the vast and accelerated emerging field of nanoscience and nanotechnology, nanofluids occupy an important area because of their potential significance facing technological challenges. Nevertheless, their commercial applications are scarce, in part because the limited understanding of the behaviour of multicomponent and multiphase systems, which requires the implication of several science areas. Among the scientific disciplines that are important in nanofluids, usually rheology plays a central role (Estellé et al., 2017; Pales et al., 2017) among others as colloid science, phase diagrams, heat transfer (Rao, 2011; Saidur et al., 2011). One of the main areas of industrial applications of nanofluids is the use of colloidal suspensions as water-based drilling fluid in oil and gas extraction and, conversely, in oil and organic solvents removal from natural waters and muds. The most recent advances in this direction seem targeted to design composite particles at the nanoscale level to improve the drilling properties of the nanofluids (see for example $\mathrm{TiO}_{2} /$ polymer nanocomposites in ref. by Sadeghalvaad and Sabbaghi, 2017).

However, suspensions of swelling clay particles still are ubiquitous in oil industry, not only for their high performance, but also for their availability in nature and environmentfriendly characteristics (Rossi et al., 1997; Murray, 2000; Ahmad et al. 2005; Okiel et al. 2011; Ganley and van Duijneveldt, 2017). The rheology of clay suspensions has been extensively studied because, depending on different variables (particle concentration, chemical composition, crystal chemistry, particle size and shape, ionic strenght, $\mathrm{pH}$, addition of stabilizing additives as surfactants and polymers), they show practically all the possible 
behaviors of non-Newtonian fluids. Usually purified (at laboratory scale) clay particles have been employed for the reological characterization of these systems (Luckam and Rossi, 1999; Duran et al., 2000; Tombacz and Szekeres, 2004; Ramos-Tejada et al. 2006 ; Abu-Jdayil 2011; Paineau et al., 2011).

In mineral deposits, different kinds of clay minerals are present (e.g. bentonite, illite, kaolite) usually mixed with other compounds (quartz, feldspar, calcite, mica). In particular, bentonite is one the clays more employed in different industrial applications (pharmaceutical, oil, nuclear), but the presence of impurities generally affects its performance because impurities modify in an uncontrolled manner the rheological properties of clay-based slurries (Penner and Lagaly, 2001). However, a precise control of the rheological properties of clay suspensions is often indispensable in industrial processes. Obviously, the purification at industrial scale requires different procedures to those employed at laboratory scale. Among the usual wet separation methods employed in chemical engineering, gravity based ones are not suitable because the gangue minerals associated to bentonite have similar densities. For this reason, those methods based on differences in particle sizes are preferable, and among them hydrocyclone is one of the more advantageous, since it exerts extra forces to induce separation and also operates at higher solid concentrations, as compared to other methods like decantation. In addition, also provides a high efficiency in a short period of time and low operating cost (Chu et al., 2004; Zhou and Liow, 2014). In fact, as Boylu and co-workers reported, when bentonites are immersed into water and scrubbed, they go into finer particles while the accompanying gangue minerals are accumulated in coarser sizes, thus giving a bimodal particle size distribution that facilitated a selective separation (Boylu et al. 2010). The hydrocyclone 
method has a further advantage for purifying calcium bentonites. Ca-bentonites oppose a high resistance to scrubbing, as compared with Na-bentonites, and frequently they require grinding process for reducing their particle size. The hydrocyclone process can be carried out using $\mathrm{Na}_{2} \mathrm{CO}_{3}$ aqueous solutions, so allowing a reduction in the $\mathrm{Ca} / \mathrm{Na}$ ratio in the exchangeable ions of the clay and improving the scrubbing of Ca-bentonites. In addition, the calcium ions precipitate as $\mathrm{CaCO}_{3}$ that can be discarded in the impurities outflow.

In this work, a raw bentonite mineral was processed by hydrocycloning, adding $\mathrm{Na}_{2} \mathrm{CO}_{3}$ solutions of different concentrations, and the resulting solid samples were characterized. Once the samples with different purity degrees were obtained, the rheological (viscous and viscoelastic) properties of bentonite suspensions with different solid concentrations were studied. The aim of this work is to identify the conditions that result in suspensions showing a strong enough elastic character, avoiding a fast sedimentation that eventually would hinder their use for recycling muds containing pollutants or similar separation processes in chemical engineering.

\section{Materials and methods}

\subsection{Bentonite mineral}

The raw bentonite was collected from Zaghouan deposit (North-Eastern of Tunisia) and the samples were air-dried and crushed by mortar and pestle and sieved through a 60-mesh screen. The selection of this Tunisian deposit was mainly due to its high yield bentonite content and its valorisation for different applications, as reported in previous works (Ayari et al., 2005; Bekri-Abbes and Srasra, 2015). This material was employed, without further 
purification (no any homoionization or cleaning), for the hydrocyclone treatment or the preparation of the raw bentonite suspensions for rheological measurements.

\subsection{Bentonite characterization}

The X-ray diffraction patterns of raw and hydrocycloned clays were obtained by a Philips $X^{\prime}$ Pert Pro diffractometer working on K $\alpha$ monochromatic radiation of copper.

The bulk chemical analysis was performed by X-ray fluorescence using an X-ray generator of 4 KW (PHILIPS Magix Pro PW-2440).

The cation exchange capacity (CEC) of the samples was determined by the Kjeldahl method (Page et al. 1982); the CEC is expressed as milli-equivalent per gram of the calcined sample.

FTIR-IR spectra were recorded on a Perkin Elmer model 597 in the $4000-400 \mathrm{~cm}^{-1}$ region using the $\mathrm{KBr}$ pellet method ( $2 \mathrm{mg}$ of sample dispersed in $200 \mathrm{mg}$ of $\mathrm{KBr}$ ).

The particle pictures were obtained by scanning electron microscopy (SEM) of variable pressure in a Zeiss SUPRA40VP apparatus.

The electrophoretic mobility (EM) measurements were carried out at $25^{\circ} \mathrm{C}$ in a Malvern Zeta-Sizer Nano SZ (Malvern Instruments, UK) in very dilute suspensions of the different samples employed. The ionic strength of the suspensions was fixed at $10^{-3} \mathrm{M} \mathrm{NaCl}$, and the EM measured $24 \mathrm{~h}$ after the preparation of the suspensions. The $\mathrm{pH}$ was readjusted immediately prior to the measurements, and the values reported correspond to the average of at least 9 data. The zeta potential was calculated by the Smoluchowski formula, assuming 
that the electric double layer thickness around the particles was very small as compared with the particle radius.

\subsection{Hydrocyclone treatment}

The purpose of the hydrocyclone treatment was the removal of impurities from the raw bentonite sample and was carried out in a laboratory scale Mozley C 750 type hydrocyclone $5 \mathrm{~cm}$ in diameter. A detailed description of the experimental setup can be seen in refs. (Özgen et al. 2009; Boylu et al. 2010; Boylu et al. 2012). Briefly, the experimental procedure was as it follows. Two different suspensions were used for hydrocycloning: i) the first one was a powder containing $1 \mathrm{~kg}$ raw bentonite and $20 \mathrm{~g}$ of $\mathrm{Na}_{2} \mathrm{CO}_{3}\left(2 \% \mathrm{Na}_{2} \mathrm{CO}_{3}\right.$ added), this powder was dispersed in $10 \mathrm{~L}$ of water for hydrocyclone treatment; ii) similar to the previous one, but with $5 \%$ of $\mathrm{Na}_{2} \mathrm{CO}_{3}$. Afterwards, the suspensions (containing $2 \%$ or $5 \%$ of $\mathrm{Na}_{2} \mathrm{CO}_{3}$ ) were kept under agitation for a period of 6 hours at room temperature. During this step, $\mathrm{Na}^{+}$ ions replaced (at least partially) the $\mathrm{Ca}^{2+}$ interlayer ions of the raw bentonite, and the removed $\mathrm{Ca}^{2+}$ ions precipitated as $\mathrm{CaCO}_{3}$. After that time interval, in which the operation of the system is considered stationary, the dispersion was pumped with a centrifuge pump to the hydrocyclone apparatus. Two discrete (overflow and underflow) products were separately obtained from the hydrocyclone (see a scheme in ref. Özgen et al., 2009). The underflow, from the bottom of the cyclone vortex $(z \approx 0, r \approx 0$ in cylindrical coordinates), contains the coarse particles, i.e., the impurities and the larger clay particles with the not scrubbed Ca-bentonite remaining. The overflow (upper part of the cyclone vortex) contains the finer particles of the raw material, i.e., the purified bentonite fraction, in which the major part consists of Na-bentonite. Finally, the samples were dried and stored for their later physico-chemical and rheological characterization. 
We refer to the different samples in this work as it follows. Sample A1: raw bentonite powder; sample A12: the purified powder obtained after hydrocycloning the raw bentonite with $2 \% \mathrm{CaCO}_{3}$ solution; sample A13: similar to $\mathrm{A} 12$ but with $5 \% \mathrm{CaCO}_{3}$.

\subsection{Preparation of the suspensions}

The above mentioned clay powders (samples A1, A12, A13) were employed for the preparation of the suspensions by dispersing $5 \% \mathrm{w} / \mathrm{v}$ or $10 \% \mathrm{w} / \mathrm{v}$ of particles in a $10^{-3} \mathrm{M}$ $\mathrm{NaCl}$ solution. Assuming a density for the bentonite particles of $2.4 \mathrm{~g} / \mathrm{cm}^{3}$ (Galindo-González at al., 2005), the solid volume fractions in the suspensions are $2.1 \%$ and $4.2 \%$ (for $5 \% \mathrm{w} / \mathrm{v}$ or $10 \% \mathrm{w} / \mathrm{v}$, respectively). The natural $\mathrm{pH}$ of the resulting suspensions was not modified (it was around $\mathrm{pH}=9$ ). The electrical conductivity of the suspensions (e.g., the suspension of sample A1 with $5 \% \mathrm{w} / \mathrm{v}$ of clay particles had a conductivity of $594 \mu \mathrm{S} / \mathrm{cm}$; for comparison the conductivity of $10^{-3} \mathrm{M} \mathrm{NaCl}$ solution is $120 \mu \mathrm{S} / \mathrm{cm}$, and for $10^{-2} \mathrm{M} \mathrm{NaCl}$ is $970 \mu \mathrm{S} / \mathrm{cm}$ ) indicates that the dispersion of the particles delivered some absorbed/adsorbed ions to the aqueous medium. Nevertheless, no any additional cleaning process (for example, successive cycles of centrifugation/redispersion in pure water) was employed because the objective was to carry out the rheological characterization in similar conditions to those used in industrial processes, when clay suspensions are employed for removing contaminants from natural waters or muds.

On the other hand, given the thixotropic behaviour of bentonite suspensions (Luckam and Rossi, 1999; Bekkour et al., 2005; Kelessidis, 2008), the protocol of suspension preparation must be precisely controlled for achieving a reliable rheological characterization. We employed three different protocols: i) stirring in a $200 \mathrm{~mL}$ flask with a magnetic bar at 
$100 \mathrm{rpm}$ during $2 \mathrm{~h}$; b) similar to the previous one, but during $24 \mathrm{~h}$; c) shaking by immersion in a ultrasonic bath during $10 \mathrm{~min}$.

\subsection{Rheological measurements}

The rheological measurements were carried out in a controlled stress rheometer (Bohlin CS10, UK), with serrated concentric cylinders (C14-Ti measuring system; inner/outer cylinder diameter $1.40 / 1.50 \mathrm{~cm}$ ) for avoiding wall slip. The temperature of the suspensions was maintained at $25{ }^{\circ} \mathrm{C}$, and the cup and bob kept in a vapour-saturated atmosphere to minimize the sample evaporation.

Two different experiments were performed: i) static or steady-state, and ii) dynamic or oscillatory. In the first one, the samples were maintained under a constant shear stress and the corresponding shear rate and viscosity (calculated as the ratio of the shear stress to the shear rate) were measured; a shear ramp between $0.15 \mathrm{~Pa}$ and $40 \mathrm{~Pa}$ was swept, with a time interval of $5 \mathrm{~s}$ between two consecutive steps. In the oscillatory tests, a sinusoidal shear stress was applied sweeping the stress amplitude from $0.15 \mathrm{~Pa}$ to $100 \mathrm{~Pa}$, and maintaining a constant frequency of $1 \mathrm{~Hz}$; the delay time between two consecutive steps was $5 \mathrm{~s}$. For each value of the applied stress amplitude, the strain amplitude, and the phase shift between the sinusoidal stress and strain were measured. From these data, the viscoelastic moduli were calculated: $G^{\prime}$ the elastic or storage modulus, and $G^{\prime \prime}$ the viscous or loss modulus.

The suspensions, after preparation by one of the above-mentioned protocols, were placed in the rheometer cell and, to ensure reliable results, the same mechanical history was built for all the samples. For this purpose, prior to the rheological measurements, the 
samples were subjected to a constant pre-shear of 50 Pa during $30 \mathrm{~s}$, followed by $120 \mathrm{~s}$ of equilibrium time.

\section{Results and discussion}

\subsection{X-ray diffraction analysis}

The results of the diffractograms of raw clay (A1), and the two samples obtained by hydrocycloning treatment ( $\mathrm{A} 12$ treated with $2 \% \mathrm{Na}_{2} \mathrm{CO}_{3}$, and $\mathrm{A} 13$ with $5 \% \mathrm{Na}_{2} \mathrm{CO}_{3}$ ) are represented in Figure 1.

\section{[Figure 1]}

The impurities in the raw material (A1 sample) are clearly showed in the spectrum of sample A1: there are important amounts of calcite (C) (reflection at $0.03 \AA$ ) and quartz (Q) (reflection at $2.28,3.34,4,25 \AA$ ), and also a low amount of kaolin (K) (reflection at 7,19 $\AA$ ). Graphs for samples $\mathrm{A} 12$ and $\mathrm{A} 13$ show that even after hydrocycloning treatment small amounts of calcite and quartz remain in the purified samples (for comparison see a similar analysis of another Tunisian clay in Boussen et al., 2015).

Concerning the clay fraction, the basal line $\left(\mathrm{d}_{001}\right)$ of the raw clay (sample $\left.A 1\right)$ that corresponds to smectite (S) appears at $15.20 \AA$, but this line moves to $12.68 \AA$ (sample $A 12$ ) and $12.48 \AA$ (sample A13) indicating a transformation from Ca-bentonite to Na-bentonite as a consequence of the replacement of $\mathrm{Ca}^{2+}$ exchange cations by $\mathrm{Na}^{+}$ones, which have a smaller atomic radius (Lu et al., 2003). On the other hand, the reflection at 1,5 $\AA$ found in all the samples indicates that the clay particles correspond to a dioctahedral smectite (Gates et al., 2002). 


\subsection{Cation exchange capacity}

The CECs of the hydrocyclone products were determined by the Kjeldhal method obtaining the following results (in meq/g units): 40 (sample A1), 56 (sample A12), and 64 (sample A13). This progressive increase of the exchange capacity can be attributed to the progressive removal of the impurities present in the raw clay by the hydrocyclone treatment. In addition, these results indirectly confirm the smaller contents of calcite and quartz in sample A13 as compared with sample A12.

\subsection{FTIR spectra of hydrocyclone products compared with raw clay}

The FTIR spectra of the hydrocylone and raw materials are represented in Figure 2. The infrared spectrum of sample A1 shows the characteristic bands of quartz and carbonate at 800 and $1420 \mathrm{~cm}^{-1}$, respectively (Page, 1982). These bands (specially that corresponding to quartz) appear with lower intensity in sample A13, indicating a smaller quantity of those impurities after hydrocycloning. The peak of Al-Al-OH stretching vibration (3450 and 904 $\mathrm{cm}^{-1}$ ) found in the spectra of all samples is typical for smectites with high amount of Al in the octahedral layer (Tunç et al., 2012). The band at $1050 \mathrm{~cm}^{-1}$ is characteristic of the valence vibration of the Si-O group of the clay network, whereas the corresponding deformation bands appear at 523 and at $466 \mathrm{~cm}^{-1}$ (Al-O-Si and Si-O-Si groups, respectively). Bands located at 1640 and $3450 \mathrm{~cm}^{-1}$ can be attributed to the deformation and valence vibrations of $\mathrm{O}-\mathrm{H}$ of water (Ayari et al., 2005).

\section{[Figure 2]}

\subsection{Morphology of the particles}


The morphology of the three samples studied is shown in the SEM pictures in Figure 3. In the photograph of sample A13 we can observe the presence of the typical morphology of layered clays, in which the particles present a stacked structure by face-to-face attachment of the bentonite platelets. A similar morphology is observed in the particles of sample A12. The particles in these hydrocycloned samples seem to have suffered scrubbing in comparison with the particles in sample $A 1$, although in all the samples rounded particles are also visible. In addition, there is a high polydispersity in the particle size, roughly ranging in the interval $100 \mathrm{~nm}-1 \mu \mathrm{m}$. Despite such polydispersity, an estimation of particle size can be provided by measuring the particle dimensions of the clay particles in a series of pictures, like those in Figure 3. The thickness $(a)$ of the bentonite platelets, even though stacked in coarse grains, in samples $A 1, A 12$, and $A 13$ are (mean \pm standard deviation): $0.37 \pm 0.15 \mu \mathrm{m}$, $0.36 \pm 0.09 \mu \mathrm{m}$, and $0.33 \pm 0.06 \mu \mathrm{m}$, respectively. The length $(L)$ of the platelets in $A 1, A 12$, and $\mathrm{A} 13$ are: $2.5 \pm 1.3 \mu \mathrm{m}, 2.4 \pm 0.8 \mu \mathrm{m}$, and $2.3 \pm 0.4 \mu \mathrm{m}$, respectively. The corresponding aspect ratios $(a / L)$ are: $0.15 \pm 0.13,0.15 \pm 0.09$, and $0.14 \pm 0.05$. These data might suggest a progressive decrease in both the particle size and the polydispersity of the platelets from A1 to A13 samples, likely due to the hydrocyclone removing of the coarse particles in A12 and A13. Nevertheless, such progressive decrease cannot be stated with the required statistical confidence because of the large values of the standard deviations of these estimations (particularly for $L$ ). This uncertainty is not rare in the case of fine particles of non-synthetic materials. However, the aspect ratio here obtained $(a / L \approx 0.14)$ is very similar to that reported in a previous work $(a / L=0,13)$ for homoionized sodium montmorillonite platelets (Ramos-Tejada et al., 2001). As a consequence, these SEM pictures give only some scarce clues about the composition of the samples, which have to be completed by the other techniques employed, as those in sections 3.1, 3.2, 3.3, and in next section 3.5. 


\section{[Figure 3]}

\subsection{Chemical composition}

The bulk chemical analysis of the clay was performed by X-ray fluorescence. The results are displayed in Table 1.

\section{[Table 1]}

The most relevant features in this analysis are as it follows. i) The decrease (in approximately one order of magnitude) in the $\mathrm{CaO} / \mathrm{Na}_{2} \mathrm{O}$ ratio between sample $\mathrm{A} 1$ and samples $A 12-A 13$, which is an indication of the effectiveness of the hydrocyclone process for removing both the $\mathrm{Ca}^{2+}$ from the clay interlayers and the calcite particles. The excessively high $\mathrm{CaO} / \mathrm{Na}_{2} \mathrm{O}$ ratio in sample $\mathrm{A} 1$, as compared with that in bentonites of high purity -see for example in ref. by Duran et al. (2000) - can be mainly assigned, in addition to Cabentinite, to the high initial calcite content of the raw clay. ii) The $\mathrm{SiO}_{2} / \mathrm{Al}_{2} \mathrm{O}_{3}$ ratio is also reduced from $\mathrm{A} 1$ to $\mathrm{A} 12-\mathrm{A} 13$ because of the removing of quartz particles favoured by the hydrocyclone treatment.

Other less relevant points in this analysis are: The high iron percentage of the samples due to the presence of $\mathrm{Fe}^{3+}$ in the crystal lattice; the relatively high values of the lost by ignition (LOI in Table 1) that can be attributed to water losses in smectite clays and, additionally, to $\mathrm{CO}_{2}$ loss from calcite; finally, note the relatively high $\mathrm{MgO}$ content that usually improves the swelling features of clays (Karagüzel et al., 2010).

\subsection{Zeta potential}


The zeta potential of the particles was determined, in diluted suspensions of the three samples, as a function of the $\mathrm{pH}$. The ionic strength of the aqueous solution was $10^{-3} \mathrm{M} \mathrm{NaCl}$ to avoid any effect of the salt concentration due to compression of the double layer thickness. The results are represented in Figure 4.

\section{[Figure 4]}

First of all, we have to mention the high standard deviation of the measurements in sample A1. This is due to the presence of different kinds of particles, in particular, the presence of quartz and calcite. The removal of these impurities by hydrocycloning allowed obtaining less disperse data in samples A12 and A13. On the other hand, the smaller zeta potential values (in absolute value) in the purified samples can be attributed to different causes. It is well known that silica and calcium carbonate particles develop a negative zeta potential in the whole pH range under study; see for example refs. by Duran et al. (2000); Moulin and Roques (2003). Specially, silica particles tend to develop a larger negative zeta potential than bentonite ones. In addition, in the acid $\mathrm{pH}$ range the edges of bentonite platelets usually bear a positive surface charge by $\mathrm{H}^{+}$adsorption; this fact favours the attachment of negative particles like silica onto the positive clay edges, so provoking the increase of the overall negative surface potential of the resulting mixed particles. The decrease in the (absolute values) zeta potentials observed for samples A12 and A13, as compared with sample $\mathrm{A} 1$, in the range of basic $\mathrm{pH}$ can be attributed to the effect of the progressive removal of quartz and calcite particles.

Finally, note that the bentonite suspensions employed in the rheological characterization below, have a pH value around 9. In such conditions it is expected that both 
the face and the edge surfaces of the clay platelets will be negatively charged by adsorption of $\mathrm{OH}^{-}$ions (potential determining ions).

\subsection{Rheological characterization}

\subsubsection{Effect of the suspension preparation protocol}

First of all, we will discuss the effect of the protocol employed for the suspension preparation. In Figure 5, the rheological results obtained in steady state (viscometry) and dynamic (oscillometry) experiments are plotted (samples A1 and A13) for the indicated protocols. In Figure 5(a), the rheograms (shear stress vs. shear rate) are plotted, and in Figure $5\left(\right.$ b) the corresponding storage modulus $\left(G^{\prime}\right)$, obtained in oscillatory measurements at constant frequency of $1 \mathrm{~Hz}$, are plotted as a function of the shear strain amplitude.

\section{[Figure 5]}

In the case of sample A1, the rheograms are very similar for the different protocols employed, and also the values of $G^{\prime}$ are not significantly different for strain amplitudes smaller than 0.2 . The sample $A 1$ practically behaves as an inelastic $\left(G^{\prime}<1 \mathrm{~Pa}\right.$; Fig. $\left.5 \mathrm{~b}\right)$ nonNewtonian fluid, probably due to the presence of large particles (size $\approx 1 \mu \mathrm{m}$, see Fig. 3a) of mixed composition (Ca-bentonite, kaolinite, quartz, calcite) with a very weak interaction among them; we will return to this point below.

In the case of the samples $A 12$ and $A 13$, the preparation protocol gave rise to different rheological behaviours. The results in Figure 5a for sample A13 (similar results found for sample A12) demonstrate that the ultrasonic (US) shaking conferred a more pronounced plastic behaviour (larger yield stress) to the suspension. This is coherent with 
the larger $G^{\prime}$ values (Fig. 5b) obtained after US. We can conclude that the better particle dispersion provoked by ultrasound, and also very likely the additional scrubbing of the stacked particles, conferred a larger elastic behaviour to the suspensions of purified samples. For this reason, the US shaking was used as the best preparation protocol for the rheological characterization described below.

\subsubsection{Steady-state measurements}

In Figure 6 the results obtained under steady shear conditions are summarized for all the suspensions studied and two different particle concentration: $5 \%$ w/v (Fig. 6a) and $10 \%$ w/v

(Fig. 6b). Similarly, in Figure 7 the corresponding viscosity curves are included. In all cases, the suspensions showed a non-Newtonian behaviour. A yield stress was required to start the flow (see intercepts in Fig. 6), which is characteristic of plastic fluids, and the viscosity decreased as the suspensions were sheared (Fig. 7), indicating a typical shear thinning response. As the concentration of the suspensions increased $(10 \% \mathrm{w} / \mathrm{v})$ and the sample became more purified (A13 > A12 > A1), a yield stress growth and a more pronounced shear thinning behaviour (viscosity drop) were observed. Nevertheless, it is worth mentioning that only in sample A13-10\%, the yield stress achieved was high enough to suggest a certain degree of attractive interactions among the particles in suspension, and only in this case a significant elastic response was expected, as we will show below in the analysis of oscillatory measurements. Furthermore, only in sample A13-10 \% the yield stress achieved was strong enough to avoid particle settling (and phase separation) in a long time period as showed in Figure 8.

From curves like these in Figures 6 it is possible to estimate different parameters that characterize the steady-state rheological response of the suspensions by fitting the post- 
yield data to the Bingham (ideal plastic behaviour) or Casson (non-ideal plastic) equations (Barnes et al., 1989):

$$
\begin{aligned}
& \sigma=\sigma_{0}+\mu \dot{\gamma} \\
& \sqrt{\sigma}=\sqrt{\sigma_{0}}+\sqrt{\mu \dot{\gamma}}
\end{aligned}
$$

where $\sigma_{0}$ is the yield stress and $\mu$ is the viscosity. These fitting parameters are included in Table 2.

\section{[Table 2]}

As observed, the general trend for $\sigma_{0}$ and $\mu$ was to increase as the solid concentration increased and the impurities of the samples were progressively removed (A13 > A12 > A1). The yield stresses estimated by Eq. (1) (Bingham model) are larger that those by Eq. (2) (Casson model) because in the Bingham fits only the data for the straight part (high shear rate) of the rheograms were considered, while for Casson all the data in the post-yield region were used. Note that, as previously mentioned, only for the suspension $\mathrm{A} 13-10 \%$ a high enough yield stress $\left(\sigma_{0} \approx 6-8 \mathrm{~Pa}\right)$ was achieved. This value is similar to that reported in a previous work ( $\sigma_{0} \approx 5 \mathrm{~Pa}$; Ramos-Tejada et al. 2001) for homoionized sodium monmorillonite suspensions ( $10 \% \mathrm{w} / \mathrm{v}, \mathrm{pH}=9$, ionic strength $\left.10^{-2} \mathrm{M} \mathrm{NaCl}\right)$. Note that in this previous work it was demonstrated that the face-to-face van der Waals interactions between particles played the dominant role leading to the plastic behaviour showed by the suspensions.

On the other hand, the shear thinning curves in Figure 7 can be characterized by fitting the data to the Sisko equation (Barnes et al., 1989): 


$$
\mu=\mu_{\infty}+K \dot{\gamma}^{n-1}
$$

where represents the viscosity at very high shear rate (the value in the high shear plateau of the viscosity curves), $K$ is the consistency, and $n$ is a potential-law parameter such as $n<1$ for shear thinning, and $n>1$ for shear thickening non-Newtonian fluids. This model is usually employed for fitting the viscosity curves in plastic fluids (where there is a significant yield stress) in the region of medium to high shear rates, i.e., when the zero shear plateau (first Newtonian region) is not available.

The parameters obtained by fitting the data in Fig. 7 to Eq. (3) are included in Table 3. The most important features are as it follows. i) The similarity of the values of (viscosities at high shear are in the order of $10 \mathrm{mPa} \cdot \mathrm{s}$ ) indicates the collapse of all the viscosity curves due to the breakage of any internal structure in the suspensions, followed by the orientation of the particles in the main flow direction at high shear, so minimizing the drag viscous force. ii) The increase in the consistency of the suspensions, which is specially high in suspension A13-10\%, once the impurities are removed by hydrocycloning, which points out that the presence of (mainly) calcite and quartz particles hinders the interactions between the bentonite particles, so reducing the order of the microstructure and consequently the consistency of the suspensions. iii) The Sisko model does not fits (no statistical significance) the viscosity data in sample A1-5\%, so confirming that this sample cannot be considered a plastic non-Newtonian fluid.

\section{[Table 3]}

\subsubsection{Oscillatory Measurements}


Finally, to complete the rheological characterization, the suspensions were stressed under an oscillatory shear, maintaining the frequency at a constant value of $1 \mathrm{~Hz}$. From the values of the imposed stress, and the corresponding strain amplitude and stress-strain phase shift, the elastic and the viscous responses were quantified by the storage $\left(G^{\prime}\right)$ and loss $\left(G^{\prime \prime}\right)$ moduli, respectively. The first one $\left(G^{\prime}\right)$ is proportional to the mean elastic power stored by the sample, and $G^{\prime \prime}$ to the power dissipated by viscous friction. The main results of these experiments are represented in Figure 9 for the suspensions with a particle concentration of $10 \% \mathrm{w} / \mathrm{v}$. Only the samples A12 and A13 developed a viscoelastic response characterized by a high enough storage modulus, while in the sample $A 1$ the modulus $G^{\prime}$ was practically negligible and, in addition, $G^{\prime} \approx G^{\prime \prime}$. The high dispersion of the data in sample $A 1$, and the very small values of $G^{\prime}$ and $G^{\prime \prime}$, are typical of inelastic non-Newtonian fluids.

\section{[Figure 9]}

In the curves for samples $\mathrm{A} 12$ and $\mathrm{A} 13$ there are two different regions. The first one is the pseudoplateau for low strain amplitude (lower than approximately $0.1 \mathrm{~Pa}$ ), which corresponds to the viscoelastic linear region (VLR) in which the applied stress is not strong enough to disrupt the internal microstructure of the samples. The second region, characterized by an abrupt decrease in the moduli, reflects a non-linear viscoelastic behaviour in which the stored and dissipated energy caused by the suspension flow are similar, once the interparticle links has been completely broken. Other interesting feature that it is worth mentioning is about the values of the moduli in the VLR. In samples A12 and A13, $G^{\prime}$ is larger than $G^{\prime \prime}$ (even one order of magnitude in the case of sample A13), which is coherent with the formation of soft gels. In fact, if we compare these results with the results in our previous work with Na-montmorillonite suspensions $(10 \% \mathrm{w} / \mathrm{v}, \mathrm{pH}=9$; see ref. 
Ramos-Tejada et al., 2001$)$ the values of $G^{\prime}$ are very similar $\left(\mathrm{G}^{\prime} \approx 40 \mathrm{~Pa}\right)$. An additional proof of the formation of soft gels in samples $A 12$ and $A 13$ is the slight increase in $G^{\prime \prime}$ at the end of the VLR (for strain amplitude approximately 0.1). This feature is characteristic of colloidal systems in which a linkage exists between the entities in the colloidal systems, being these particles or polymer chains. An example of this slight increase of $G^{\prime \prime}$, in a gel consisting of solid particles crosslinked by polymer chains, can be seen in a recent work (BonhomeEspinosa et al., 2017). Obviously, none of these characteristics is observed in the suspension A1, which can be taken as a confirmation of the lack of relevant interaction between particles in this system.

A final question arises about the nature and intensity of the interactions that favour the gel formation in the hydrocycloned samples. We have to refer to our previous work with Na-Montmorillonite (Duran et al. 2000). In this work it was demonstrated that at $\mathrm{pH}=9$ the face-to-face and face-to-edge electrostatic repulsions between clay platelets in $\mathrm{Na}$ montmorillonite imposed an energy barrier with a maximum at an interparticle distance $H \approx$ $5 \mathrm{~nm}$. Nevertheless, the electrostatic repulsion suffered a sharp (practically exponential) decay down to zero at $H \approx 20 \mathrm{~nm}$. On the contrary, the van der Waals (VdW) attraction between pairs of particles has a long-range decay (the VdW energy of interaction depends on $\mathrm{H}^{-2}$ ). As a consequence, the $\mathrm{VdW}$ attraction favoured the flocculation among the particles in suspension; our estimation in that previous work was that the total energy of interaction between the different Na-montmorillonite surfaces (at the minimum of the potential well, at $H \approx 12 \mathrm{~nm}$ ) varied approximately between $-100 \mathrm{kT} / \mu \mathrm{m}^{2}$ (face-to-face) and $-150 \mathrm{kT} / \mu \mathrm{m}^{2}$ (faceto-edge). Obviously, as the particle aspect ratio (= particle length/thickness) is roughly 10 in the present work (see Fig. 3c), the surface area of the faces is considerably higher than that 
of edges, and consequently the most favoured flocculation is face-to-face, although the faceto-edge links must induce the formation of the typical house of cards microstructure in bentonite suspensions. Certainly, this structure has to be enough opened, composed by loose flocculi that occupy all the suspension volume, because in suspensions of nonspherical particles with a solid volume fraction of around $5 \%$ (taking a density for clay particles of $2 \mathrm{~g} / \mathrm{cm}^{3}$, particle concentration $10 \% \mathrm{w} / \mathrm{v}$ ), the Bingham yield stress reaches a value of only $\sigma_{0} \approx 8 \mathrm{~Pa}$ (Table 2 ) and the elastic modulus of $G^{\prime}=37 \mathrm{~Pa}$ (Fig. 9). Nevertheless, and this point is important for the industrial applications of hicrocycloned bentonites, such values of the rheological quantities are high enough to avoid phase separation by gravitational settling and, consequently, to ensure the availability of stable suspensions.

\section{Conclusions}

Hydrocyclone is a wet separation process usually employed at industrial scale to operate with solid materials that contain grains of several minerals with different particle sizes. This method seems especially useful when the different minerals have similar densities and other methods, based in gravitational forces, are not efficient. There are a number of reasons that make hydrocyclone operation appropriate for clay minerals like bentonites. The first one is its efficiency (at low cost) to separate bentonite particles from the usual gangues mixed with this clay, as calcite, quartz, and kaolinite, among other silicates. The second one is the possibility of using aqueous solutions that allow the change of the interlayer (exchange) clay ions for other ions more convenient from the point of view of the application. In this work, sodium carbonate solutions have been employed for substituting the calcium ions in the original Ca-bentonite for the sodium one. The carbonate ions in the liquid phase of the hydrocyclone play an additional role because they allow the precipitation, and posterior 
separation, of the exchanged $\mathrm{Ca}^{2+}$ in the form of calcium carbonate particles. The third advantage of the hydrocyclone in bentonite clays resides in the wet scrubbing of the stacked platelets present in the raw clay particles. This disaggregation has low efficiency in hard Cabentonite aggregates but results easier in $\mathrm{Na}$-bentonite, something that valorises even more the above-mentioned ion exchange provoked by the addition of $\mathrm{Na}_{2} \mathrm{CO}_{3}$ solution.

We have used different methods for the characterization of the bentonite powder obtained by hydrocycloning. Results of all these methods are consistent with the effectiveness of hydrocycloning for removing the impurities present in the raw material (mainly calcite and quartz), and also with the progressive change of the original Ca-bentonite for Na-bentonite, by using more concentrated $\mathrm{Na}_{2} \mathrm{CO}_{3}$ solutions.

In the rheological characterization of non-Newtonian suspensions, the protocol used for the particle dispersion in the liquid medium plays a central role because of the usual time dependent behaviour (thixotropy) of these complex fluids. We have demonstrated in this work that the more efficient method for the dispersion of the bentonite particles is ultrasonic shaking, very likely because the scrubbing of the stacked clay platelets, which should favour the formation of stable (non-settling) gels, in the case of using samples with small proportion of impurities.

We have also shown that the presence of calcite and quartz particle impurities results in non-Newtonian suspensions with negligible yield stress and elastic response and, as a consequence, these suspensions suffer of fast gravitational settling, what represents a serious inconvenient for their industrial uses as drilling fluids or for removing pollutants in muds. Additionally, we have demonstrated that it is possible to avoid phase separation when: i) the hydrocyclone process is efficient enough -in the present work by using a $5 \%$ 
$\mathrm{Na}_{2} \mathrm{CO}_{3}$ solution in the cyclone; and ii) the volume fraction of bentonite particles in the suspensions is at least of the order of $5 \%$ (or $10 \% \mathrm{w} / \mathrm{v}$ ). Only under such conditions the suspensions have a plastic behaviour with a high enough dynamic yield stress (about 5-8 Pa) and elastic response (storage modulus around $40 \mathrm{~Pa}$ ), so that a gel consistency is provided to the clay suspensions.

The formation of soft gels with Na-bentonite at the natural $\mathrm{pH}$ of the suspensions (well into the range of basic $\mathrm{pH}$ ) is possible, even when an electrostatic repulsion (both faces and edges bear negative charge) between the platelet surfaces exists. This is because the long-range van der Waals attractions makes possible the formation of a microstructure with loose flocculi that occupy all the volume of the suspensions.

When bentonite clays are employed in the conditions explained above, it is possible to have colloidal suspensions with appropriate stability against gravitation settling, for industrial purposes, in which the use of additional stabilizing additives (surfactants, polymers, composite materials) can be unneeded or at least minimized.

\section{Acknowledgments}

This study was supported by project FIS2013-41821-R (Plan Nacional de Investigación Científica, Desarrollo e Innovación Tecnológica, Ministerio de Economía y Competitividad, Spain, co-funded by ERDF, European Union). M. M. A. acknowledges financial support from Tunisian Goverment (fellowship program) and UE (Erasmus program) for her stays in the University of Granada. 


\section{References}

B. Abu-Jdayil, Int. J. Miner. Process. 98, 208 (2011)

A. L. Ahmad, S. Sumathi, and B. H. Hameed, Chem. Eng. J. 108, 179 (2005)

F. Ayari, E. Srasra, and M. Trabelsi-Ayadi, Desalination 185, 391 (2005)

H. A. Barnes, J. F. Hutton and K. Walters, An Introduction to Rheology, Elsevier, Amsterdam. (1989).

K. Bekkour, M. Leyama, A. Benchabane, and O. Scrivener, J. Rheology 49, 1329 (2005)

I. Bekri-Abbes and E. Srasra, Mater. Sci. Semicond. Process. 40, 543 (2015)

A. B. Bonhome-Espinosa, F. Campos, I. A. Rodriguez, V. Carriel, J. A. Marins, A. Zubarev, J. D.

G. Duran, and M. T. Lopez-Lopez, Soft Matter 13, 2928 (2017)

S. Boussen, D. Sghaier, F. Chaabani, B. Jamoussi, S. B. Messaoud, and A. Bennour, Appl. Clay Sci. 118, 344-353 (2015)

F. Boylu, K. Çinku, F. Esenli, and M. S. Çelik, Int. J. Miner. Process. 94, 196 (2010)

F. Boylu, R. Hojiyev, G. Ersever , Y. Ulcay, and M. S. Çelik, J. Sep. Sci. Technol. 47, 842 (2012)

L. Y. Chu, W. Yu, J. Wang, X. T. Zhou, W. Chen, and G. Q. Dai, G.-Q., Chem. Eng. Process. Process Intensif. 43, 1441 (2004)

J. D. G. Duran, M. M. Ramos-Tejada, F. J. Arroyo, and F. Gonzalez-Caballero, J. Colloid Interf. Sci. 229, 107 (2000)

P. Estellé, F. Fraïsse, A. Audfray, T. Maré, and Cong Tam Nguyen, J. Nanofluids 6, 213 (2017)

C. Galindo-González, J. de Vicente, M. M. Ramos-Tejada, M. T. López-López, F. GonzálezCaballero, and J. D. G. Durán, Langmuir 21, 4410 (2005)

W. J. Ganley and J. S. van Duijneveldt, Langmuir 33, 1679 (2017)

W. Gates, J. Anderson, M. Raven, and G. Churchman, Appl. Clay Sci. 20, 189 (2002) 
C. Karagüzel, T. Çetinel, F. Boylu, K. Çinku, and M. S. Çelik, Appl. Clay Sci. 48, 398 (2010)

V. C. Kelessidis, Energy Source. Part A, 30, 1729 (2008)

X. Lu, X. Cui, and M. Song, Miner. Eng. 16, 1303 (2003)

P. F. Luckham and S. Rossi, Adv. Colloid Interfac. 82, 43 (1999)

P. Moulin and H. Roques, J. Colloid Interface Sci. 261, 115 (2003)

H. H. Murray, App. Clay Sci.17, 207 (2000)

K. Okiel, M. El-Sayed, and M. Y. El-Kady, Egypt. J. Petrol. 20, 9 (2011)

S. Özgen, A. Yıldız, A. Çalışkan, and E. Sabah, Appl. Clay Sci. 46, 305 (2009)

A. L. Page, R. H. Miller and D. R. Keeney, Kjeldhal N2 analysis. Methods of soil analysis, Soil Science Society of America INC. Publisher, Madison, Wisconsin (1982)

A. R. Pales, E. N. Kinsey, C. Li, L. Mu, L. Bai, H. M. Clifford, and C. J. G. Darnault, J. Nanofluids 6, 795 (2017)

E. Paineau, L. J. Michot, I. Bihannic, and C. Baravian, Langmuir 27, 7806 (2011)

D. Penner and G. Lagaly, App. Clay Sci. 19, 131 (2001)

M. M. Ramos-Tejada, F. J. Arroyo, R. Perea, J. D. G. Duran, J. Colloid Interf. Sci. 235, 251-259 (2001)

M. M. Ramos-Tejada, C. Galindo-González, R. Perea, and J. D. G. Duran, J. Rheology 50, 995 (2006)

Y. Rao, Particuology 8, 549 (2010)

S. Rossi, P. F. Luckham, S. Zhu, B. J. Briscoe, and Th.F. Tadros, Rev. I. Fr. du Petrol. 52, 199 (1997)

M. Sadeghalvaad and S. Samad, J. Nanofluids 6, 205 (2017)

R. Saidur, K.Y. Leong, and H. A. Mohammad, Renew. Sust. Energ. Rev. 15, 1646 (2011)

E. Tombácz, and M. Szekeres, App. Clay Sci. 27, 75 (2004) 
S. Tunç, O. Duman, and B. Kanci, Colloid. Surface. A 398, 37 (2012)

G. Zhu and J. Liow, J., Chem. Eng. Sci. 111, 94 (2014) 


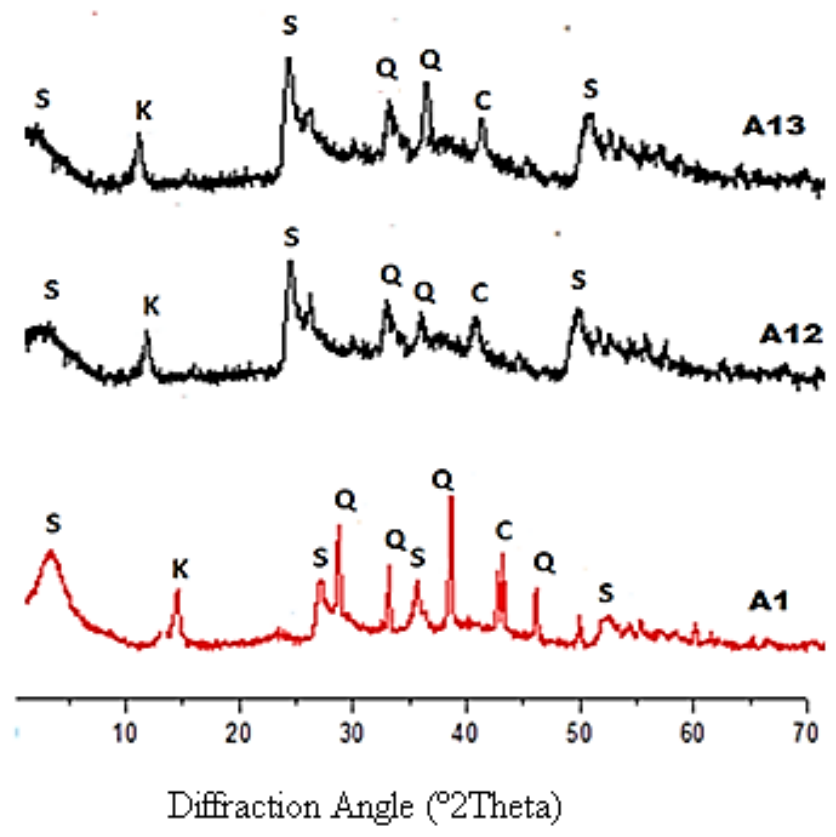

Figure 1. X-ray diffraction pattern of the raw sample (A1), hydrocycloned sample with $2 \%$ $\mathrm{Na}_{2} \mathrm{CO}_{3}(\mathrm{~A} 12)$, and hydrocycloned sample with $5 \% \mathrm{Na}_{2} \mathrm{CO}_{3}(\mathrm{~A} 13)$. S: smectite (bentonite), $\mathrm{K}$ : kaolinite, Q: quartz, C: calcite. 


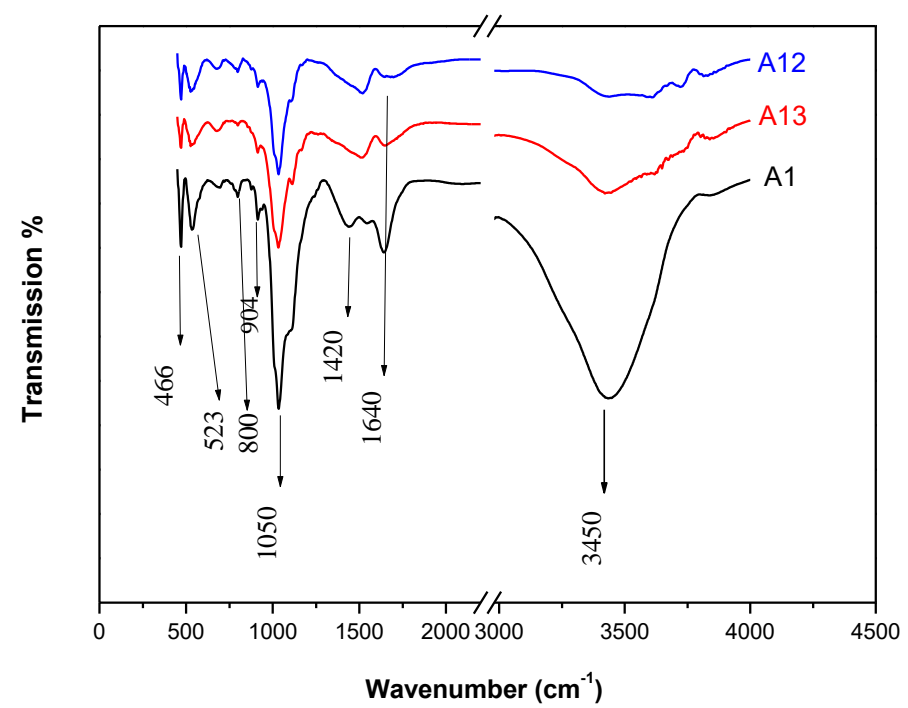

Figure 2. FTIR spectra of the raw clay (A1), and hydrocycloned samples A12 and A13. 
a)

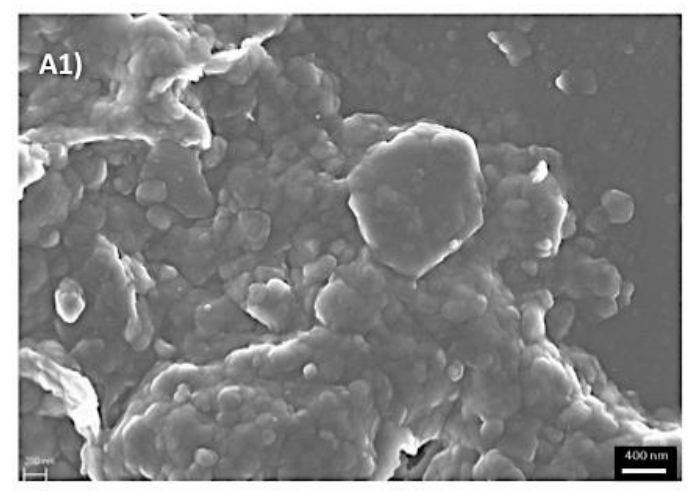

b)

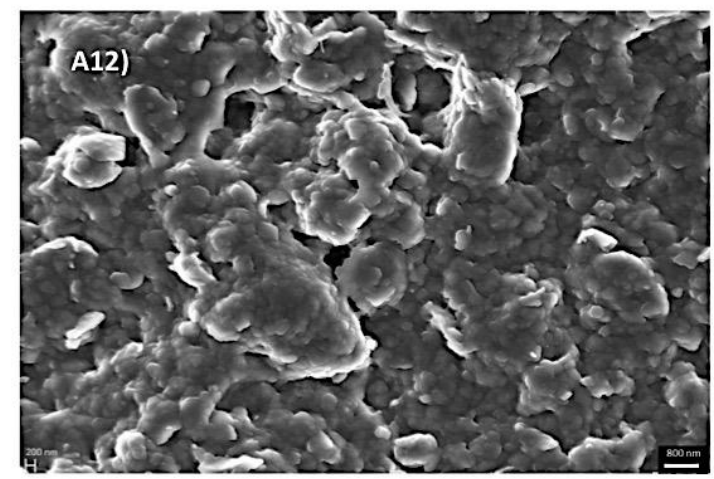

c)

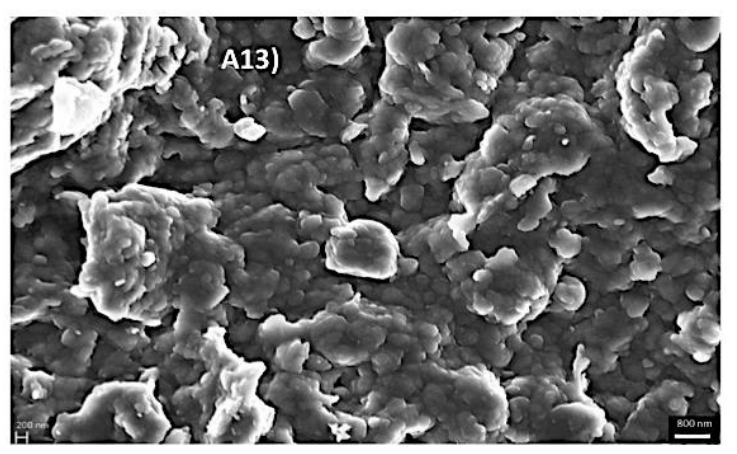

Figure 3. SEM pictures of the bentonite samples: a) sample $A 1$, bar length $400 \mathrm{~nm}$; b) sample A12, bar length $800 \mathrm{~nm}$; c) sample A13, bar length $800 \mathrm{~nm}$. 


\begin{tabular}{|c|c|c|c|}
\hline Compound & Sample A1 & Sample A12 & Sample A13 \\
\hline $\mathrm{SiO}_{2}$ & 50,49 & 50,09 & 49,28 \\
\hline $\mathrm{Al}_{2} \mathrm{O}_{3}$ & 20,48 & 21,34 & 20,82 \\
\hline $\mathrm{Fe}_{2} \mathrm{O}_{3}$ & 8,20 & 7,15 & 6,98 \\
\hline $\mathrm{CaO}$ & 3,33 & 3,03 & 3,74 \\
\hline $\mathrm{MgO}$ & 2,67 & 2,79 & 2,80 \\
\hline $\mathrm{Na}_{2} \mathrm{O}$ & 0,19 & 1,15 & 1,94 \\
\hline $\mathrm{K}_{2} \mathrm{O}$ & 2,04 & 2,05 & 2,02 \\
\hline $\mathrm{P}_{2} \mathrm{O}_{5}$ & 0,20 & 0,18 & 0,11 \\
\hline $\mathrm{TiO}_{2}$ & 1,14 & 1,11 & 1,08 \\
\hline $\mathrm{MnO}$ & 0,03 & 0,02 & 0,02 \\
\hline LOI & 11,15 & 10,33 & 10,56 \\
\hline $\mathrm{CaO} / \mathrm{Na}_{2} \mathrm{O}$ & 17.5 & 2.63 & 1.93 \\
\hline $\mathrm{SiO}_{2} / \mathrm{Al}_{2} \mathrm{O}_{3}$ & 2,46 & 2,35 & 2,36 \\
\hline
\end{tabular}




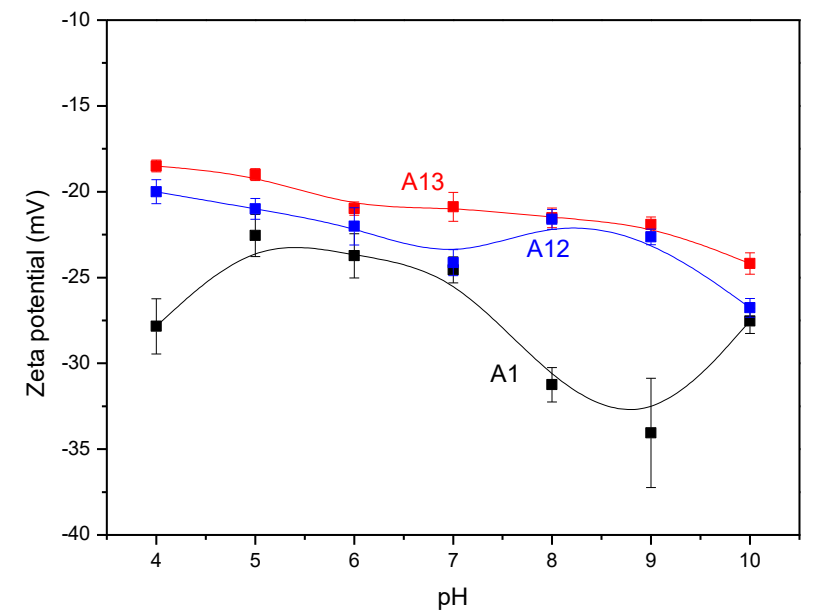

Figure 4. Zeta potential of the particles in suspension as a function of $\mathrm{pH}$ for the three samples studied. Ionic strength $10^{-3} \mathrm{M} \mathrm{NaCl}$. 
a)

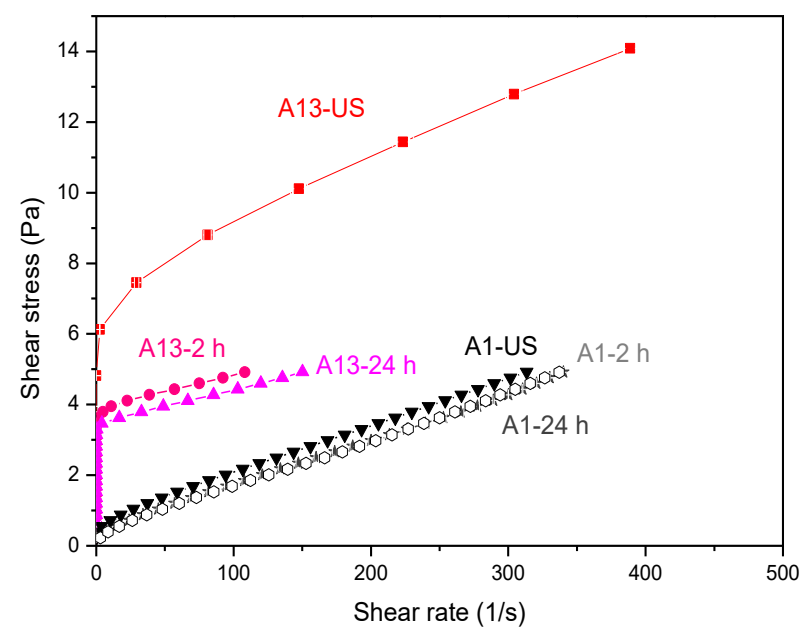

b)

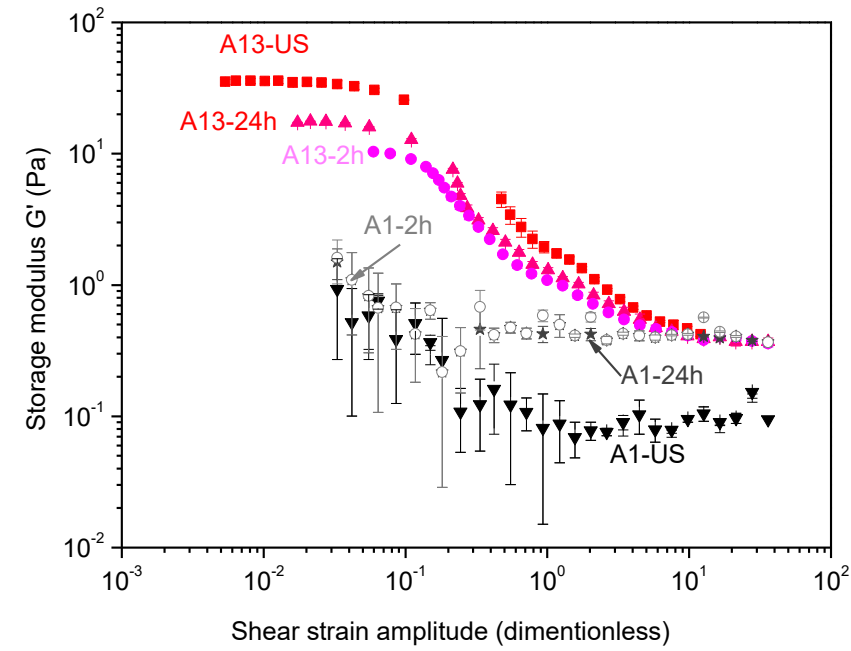

Figure 5. a) Rheograms of the suspensions of samples $A 1$ and $A 13$ (particle concentration 10 $\% \mathrm{w} / \mathrm{v}$ ) obtained after different preparation protocols. US: ultrasonic shaking during $10 \mathrm{~min}$; $2 \mathrm{~h}$ : mechanical stirring during $2 \mathrm{~h} ; 24 \mathrm{~h}$ : mechanical stirring during $24 \mathrm{~h}$. b) Storage modulus as a function of the strain amplitude (oscillatory shear, constant frequency $1 \mathrm{~Hz}$ ) for the same samples and preparation protocols indicated in (a). 
a)

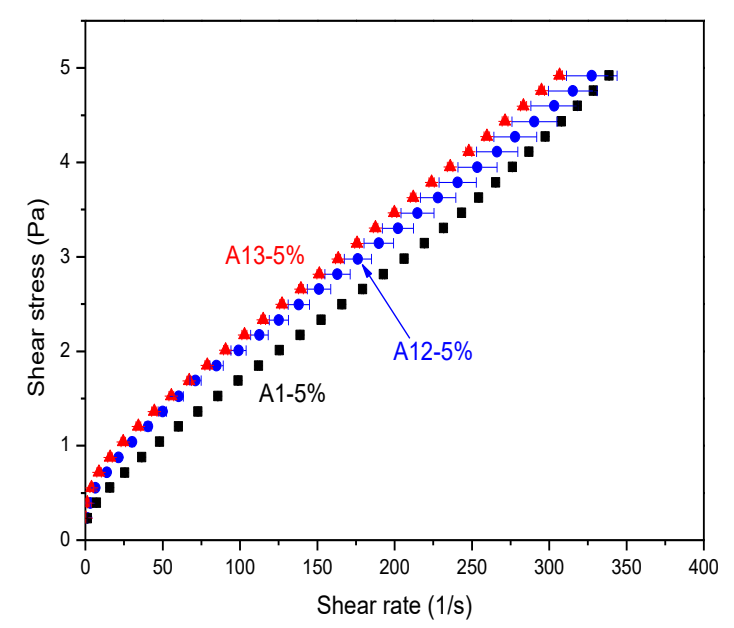

b)

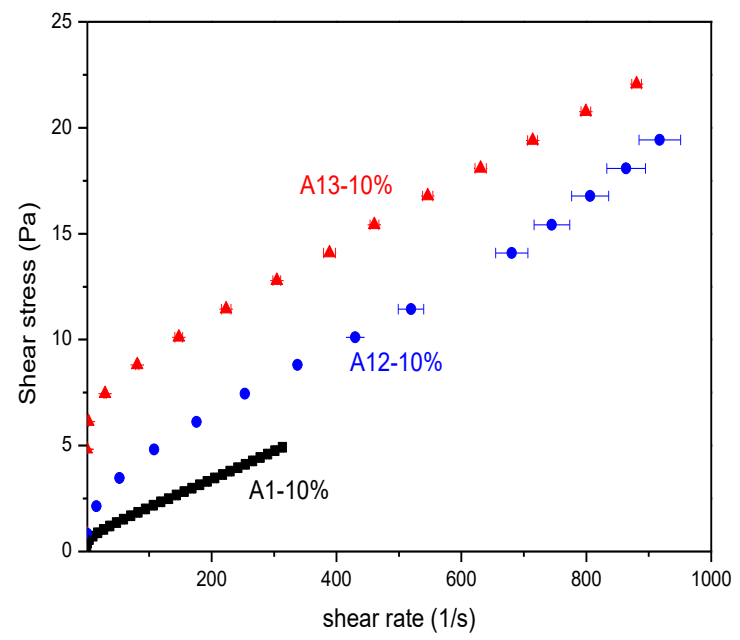

Figure 6. Rheograms of the clay suspensions for particle concentrations of $5 \% \mathrm{w} / \mathrm{v}(\mathrm{a})$ and $10 \% \mathrm{w} / \mathrm{v}(\mathrm{b})$. 
a)

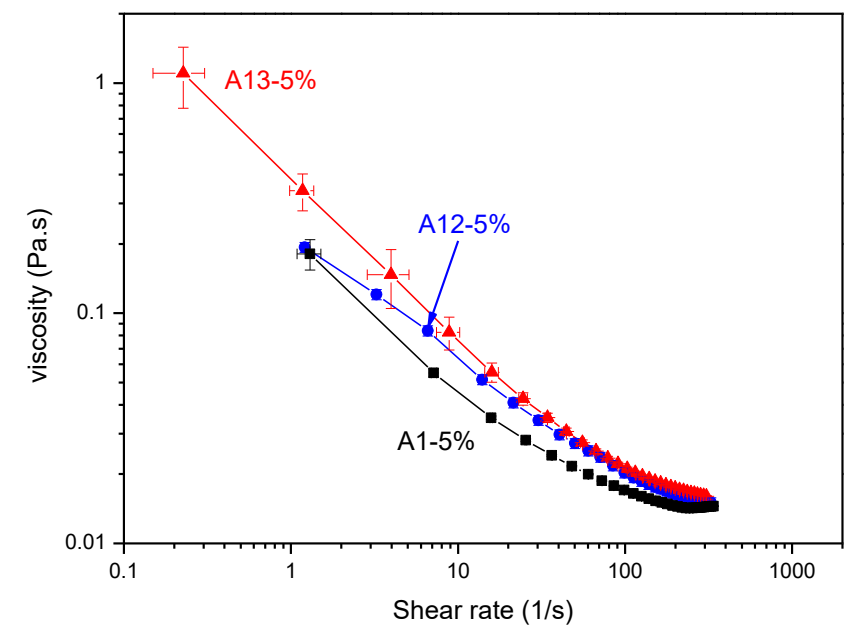

b)

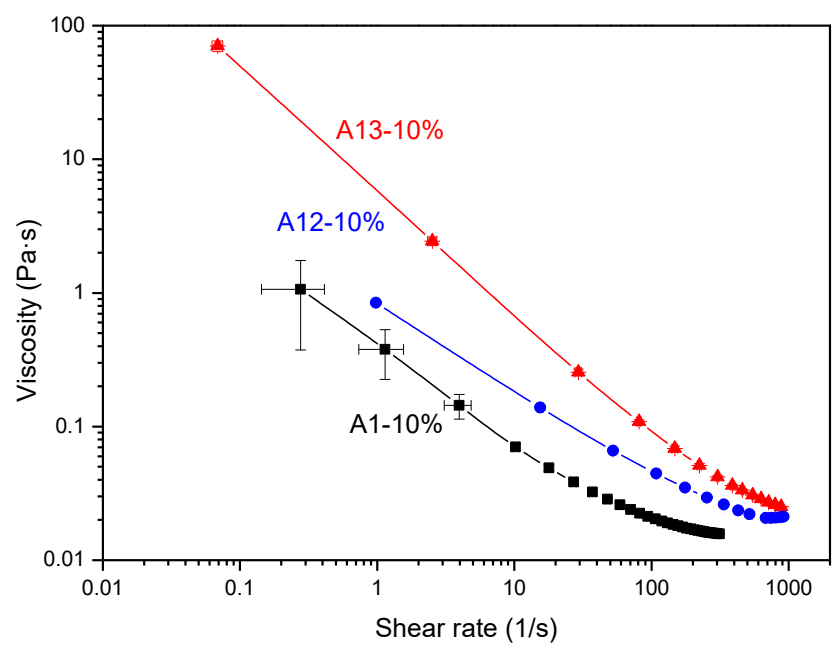

Figure 7. Viscosity as a function of shear rate obtained from the rheograms in Fig. 6 . The viscosity is calculated as the ratio shear stress/shear rate for each experimental point in Fig. 6. 

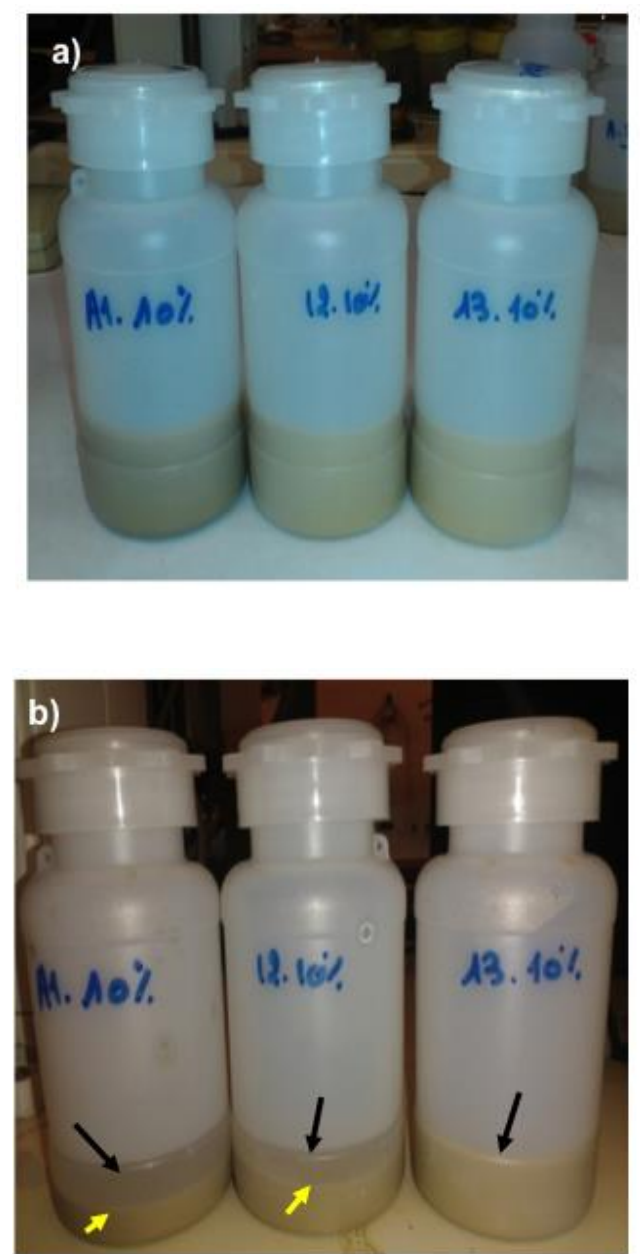

Figure 8. a) Clay suspensions just prepared ( $10 \% \mathrm{w} / \mathrm{v}$, from left to right samples $A 1, A 12$, A13). b) The same suspensions after gravitational settling during 10 days; black arrows: liquid-air interface; yellow arrows: solid (sediment)-liquid interface. 
Table 2. Parameters characterizing the steady-state behaviour of the suspensions obtained by fitting the data in Fig. 6 to Bingham and Casson equations. $\sigma_{0}$ yield stress; $\mu$ viscosity, and $r^{2}$ coefficient of determination.

\begin{tabular}{|c|c|c|c|c|c|c|}
\hline \multirow[t]{2}{*}{ Sample } & \multicolumn{3}{|c|}{ Particle concentration $5 \% \mathrm{w} / \mathrm{v}$} & \multicolumn{3}{|c|}{ Particle concentration $10 \% \mathrm{w} / \mathrm{v}$} \\
\hline & $\begin{array}{c}\sigma_{0}(\mathrm{~Pa}) \\
\text { Bingham } \\
\text { Casson }\end{array}$ & $\begin{array}{c}\mu(\mathrm{Pa} \cdot \mathrm{s}) \\
\text { Bingham } \\
\text { Casson }\end{array}$ & $\begin{array}{c}r^{2} \\
\text { Bingham } \\
\text { Casson }\end{array}$ & $\begin{array}{c}\sigma_{0}(\mathrm{~Pa}) \\
\text { Bingham } \\
\text { Casson }\end{array}$ & $\begin{array}{c}\mu(\mathrm{Pa} \cdot \mathrm{s}) \\
\text { Bingham } \\
\text { Casson }\end{array}$ & $\begin{array}{c}r^{2} \\
\text { Bingham } \\
\text { Casson }\end{array}$ \\
\hline A1 & $\begin{array}{c}0.37 \pm 0.02 \\
0.341 \pm 0.022\end{array}$ & $\begin{array}{c}0.01305 \pm 0.00012 \\
0.01317 \pm 0.00011\end{array}$ & $\begin{array}{l}0.99781 \\
0.99802\end{array}$ & $\begin{array}{l}0.72 \pm 0.01 \\
0.60 \pm 0.03\end{array}$ & $\begin{array}{l}0.01341 \pm 0.00005 \\
0.01395 \pm 0.00019\end{array}$ & $\begin{array}{l}0,99964 \\
0.99561\end{array}$ \\
\hline A12 & $\begin{array}{l}0.68 \pm 0.01 \\
0.58 \pm 0.03\end{array}$ & $\begin{array}{l}0.01294 \pm 0.00009 \\
0.0134 \pm 0.000019\end{array}$ & $\begin{array}{l}0.99874 \\
0.99423\end{array}$ & $\begin{array}{l}1.47 \pm 0.51 \\
0.84 \pm 0.27\end{array}$ & $\begin{array}{c}0.0204 \pm 0.0006 \\
0.02035 \pm 0.00005\end{array}$ & $\begin{array}{c}0,9856 \\
0.99478\end{array}$ \\
\hline A13 & $\begin{array}{l}0.72 \pm 0.01 \\
0.62 \pm 0.03\end{array}$ & $\begin{array}{r}0.0137 \pm 0.00008 \\
0.01422 \pm 0.00021\end{array}$ & $\begin{array}{l}0.99917 \\
0.99393\end{array}$ & $\begin{array}{c}7.67 \pm 0.06 \\
5.8 \pm 0.7\end{array}$ & $\begin{array}{c}0.0165 \pm 0.00009 \\
0.0195 \pm 0.0014\end{array}$ & $\begin{array}{l}0,99955 \\
0.92952\end{array}$ \\
\hline
\end{tabular}


Table 3. Parameters that characterize the shear thinning behaviour of the suspensions estimated by fitting the data in Figure 7 to the Sisko model (Eq. 3).

\begin{tabular}{lcccc}
\hline Sample & $(\mathrm{Pa} \cdot \mathrm{s})$ & $K\left(\mathrm{~Pa} \cdot \mathrm{s}^{\mathrm{n}}\right)$ & $n(-)$ & $r^{2}$ \\
\hline A1-5\% & $0.01427 \pm 0.00011\left(^{*}\right)$ & $1.3 \pm 1.1\left(^{*}\right)$ & $-0.63 \pm 0.25\left(^{*}\right)$ & $0.10642\left(^{*}\right)$ \\
\hline A12-5\% & $0.0090 \pm 0.0004$ & $0.225 \pm 0.006$ & $0.359 \pm 0.013$ & 0.99285 \\
\hline A13-5\% & $0.01254 \pm 0.00013$ & $0.4245 \pm 0.022$ & $0.1570 \pm 0.014$ & 0.99594 \\
\hline A1-10\% & $0.01451 \pm 0.00006$ & $0.74 \pm 0,04$ & $-0.891 \pm 0.024$ & 0.9971 \\
\hline A12-10\% & $0.0106 \pm 0.0004$ & $0.84 \pm 0,03$ & $0.31 \pm 0,01$ & 0.99884 \\
\hline A13-10\% & $0.01506 \pm 0.00007$ & $5.73 \pm 0.04$ & $0.0632 \pm 0.0017$ & 0.99985
\end{tabular}

(*) Estimates with very low statistical confidence 


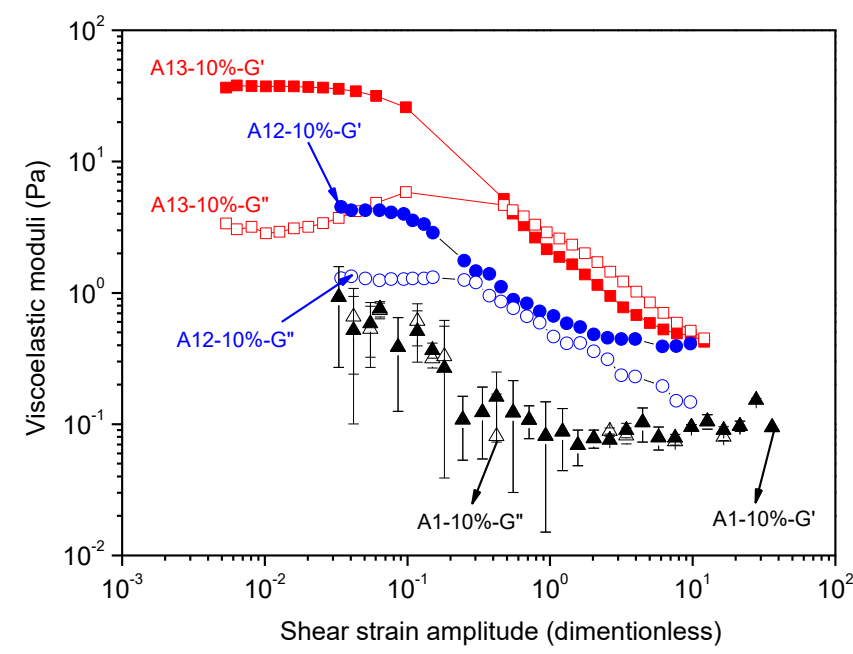

Figure 9. Viscoelastic moduli as a function of the strain amplitude, obtained under oscillatory stress at a constant frequency of $1 \mathrm{~Hz}$. Suspension concentration $10 \% \mathrm{w} / \mathrm{v}$. Solid symbols: storage modulus $G^{\prime}$. Open symbols: loss modulus $G^{\prime \prime}$. 